First author:

Dov Zazkis

Arizona State University

School of mathematical and statistical science

Second author:

Keith Weber

Rutgers University

Graduate school of education

Third author

Juan Pablo Mejía-Ramos

Rutgers University

Graduate school of education

Dov Zazkis, Ph.D.

Visiting Assistant Professor

Department of Mathematics

Oklahoma State University

E: zazkis@gmail.com

P: (405)744-5541 


\section{Two proving strategies of highly successful mathematics majors}

Abstract. We examined the proof-writing behaviors of six highly successful mathematics majors on novel proving tasks in calculus. We found two approaches that these students used to write proofs, which we termed the targeted strategy and the shotgun strategy. When using a targeted strategy students would develop a strong understanding of the statement they were proving, choose a plan based on this understanding, develop a graphical argument for why the statement is true, and formalize this graphical argument into a proof. When using a shotgun strategy, students would begin trying different proof plans immediately after reading the statement and would abandon a plan at the first sign of difficulty. The identification of these two strategies adds to the literature on proving by informing how elements of existing problem-solving models interrelate.

Key words: Problem-solving; Proof; Undergraduate mathematics education; proving strategies. 


\section{Introduction}

One of the central purposes of many university advanced mathematics courses is to increase mathematics majors' abilities to write proofs (Weber, 2001). Unfortunately, even after completing these courses, many research studies indicate that proof writing remains a persistent difficulty for mathematics majors. In many studies, mathematics majors were asked to complete a set of proving tasks; in each case, they collectively proved substantially fewer than half of the statements assigned to them (e.g., Alcock \& Weber, 2010; Hart, 1994; Iannone \& Inglis, 2010; Ko \& Knuth, 2009; Moore, 1994; Weber, 2001; Weber \& Alcock, 2004).

There has been substantial research on mathematics majors' difficulties with writing proofs (see Selden \& Selden, 2008, for a comprehensive review), which include epistemological, logical, conceptual, and strategic difficulties. By epistemological difficulties we mean that students need to be persuaded by the same types of arguments that mathematicians consider convincing (Harel \& Sowder, 1998, 2007). Misalignments between what mathematicians find convincing and what students find convincing may lead to students' difficulties with both production and comprehension of normatively correct proofs. In particular, the goals that students set for themselves during the proving process may be inconsistent with what their course instructor expects of them. By logical difficulties we mean difficulties with formal logic. These include students' understanding of what forms of logical inferences are permissible (e.g., modus tollens) (Weber \& Alcock, 2005), students' understandings of multiply qualified statements (Zandieh, Roh, \& Knapp, 2014) and how one can logically structure a proof (Selden \& Selden, 1995; Savić, 2011). Conceptual difficulties are difficulties with students' understanding of 
concepts and definitions (Hart, 1994; Tall \& Vinner, 1981). These difficulties may lead students to make false inferences or leave students unable to make a critical deduction that is needed to write a proof. Finally, by strategic difficulties we are referring to issues with students' problem-solving approaches. These include which strategies students use to generate a proof, when and how they choose to abandon particular strategies, and how they decide which strategies are worth pursuing (Anderson, Boyle, \& Yost, 1986; Schoenfeld, 1985; VanSpronsen, 2008; Weber, 2001). Although all of these types of difficulties are important, in this work we focus on the last of these categories, students' proving strategies.

One approach to identifying the strategies needed to construct proofs is to carefully study the behavior of those who are proficient at proof writing. There has been limited work in this area. A first body of work has examined the proof-writing behavior of mathematicians. Using an expert-novice research paradigm, Weber (2001) identified proof writing heuristics that mathematicians used in abstract algebra that undergraduates appeared to lack. Lockwood et al. (2012) studied the ways in which mathematicians used examples to solve problems and write proofs. Samkoff, Lai, and Weber (2012) explored how mathematicians used diagrams when writing a proof. This focus on mathematicians provides valuable insight into how proofs may be successfully written, but it has an important limitation. Mathematicians' proving strategies might rely on experiences and understandings that most undergraduates may lack. If so, exposing students to these strategies without these corresponding experiences may lead to naïve application of the strategies, which can be counterproductive (cf., Reif, 2008).

A second set of studies has examined the strategies of students who wrote proofs 
successfully. Gibson (1998) investigated how undergraduates' use of diagrams enabled them to overcome impasses when writing proofs in real analysis. Sandefur, Mason, Stylianides, and Watson (2013) studied how considering examples aided two groups of undergraduates on a specific task in number theory. The goal of this paper is to contribute to this second set of studies. To do so, we studied the proof-writing behavior of highly successful mathematics majors. Specifically, we examined six highly successful mathematics majors who performed well, both in their proof-oriented mathematics courses and in proving tasks that we assigned to them in this study in order to glean their proof strategies.

\section{Theoretical framing}

In this paper, we view proof writing as a problem-solving task in which the student is given a statement to prove and has the goal of producing a deductive argument that establishes the statement to be proven while conforming with mathematical norms (Furinghetti \& Morselli, 2007; Weber, 2005). Rather than problematize the personal goal that students are trying to achieve or the knowledge base they have, we are interested in how the students marshal the resources they have to write proofs. This suggests two strategic difficulties that students may have. The first strategic difficulty involves students' choice of approach. The set of potential approaches to most proving tasks are numerous, but only a fraction of these approaches are likely to be useful. Students may choose a wrong approach and hence not produce a proof (Anderson, Boyle, \& Yost, 1986; Schoenfeld, 1985; Weber, 2001). Hence, how students choose which approach to implement is of particular importance when studying their proof generation strategies. 
The second related consideration concerns when students reach an impasse and do not know how to proceed. This can occur when a student reaches a dead end when implementing one approach or simply when the student cannot spontaneously generate any way to approach the task. That is, a student may feel that helshe can no longer take actions that are potentially productive to further progress with their current plan (e.g., Moore, 1994; Schoenfeld, 1980; Weber, 2001). Hence, an important strategic consideration involves examining how students identify when an impasse has been reached and what they do in an effort to overcome it.

\subsection{Understanding and planning}

The literature suggests several ways of negotiating the interplay between strategy choice and impasses. A common suggestion in the literature is that when students encounter a problem-solving task, they should first spend time working to understand the problem and then carefully choose a plan to solve the problem (Polya, 1945; Schoenfeld, 1985). More recent work has demonstrated a complicated interaction between the stages of understanding, planning, and implementing a plan; indeed, expert problem solvers may form a plan to understand some aspect of a problem that is confusing to them (Carlson \& Bloom, 2005). But most models of expert mathematical problem-solving agree that one spends at least some time initially trying to understand a problem before solving it. Students tend not to do this and this is one account given to explain their difficulties with problem-solving (Schoenfeld, 1985).

\subsection{Metacognition and monitoring}

Schoenfeld (1985) observed that expert problem solvers not only carefully chose their problem-solving plan but they also monitored their progress in applying this plan. 
In Schoenfeld's studies, mathematicians would repeatedly ask themselves monitoring questions such as, "what is this plan trying to achieve?" and "do I think I can achieve these goals?", using their responses to guide their actions, including switching plans if necessary. In contrast, students tended to be more single-minded, with the result being that they failed to solve a problem since their efforts were entirely comprised of following a plan that was doomed from the start (Schoenfeld, 1987). DeFranco (1996) expanded upon Schoenfeld's results in his study of PhD Mathematicians. DeFranco compared problem-solving experts (mathematicians who had attained acclaim as researchers) with domain experts (mathematicians who did not attain such eminence). DeFranco found that the latter group was less successful on problem-solving tasks than the former, with the domain experts' lack of success on problem-solving tasks being due, in part, to their lack of monitoring and metacognition.

\subsection{Informal representations}

Although proofs in advanced mathematical contexts are usually written deductively in a verbal-symbolic syntax, some mathematics educators have remarked that the processes used to create these proofs may rely on less formal representations (e.g., Garuti, Boero, \& Lamut, 1998; Lockwood et al., 2013; Sandefur, Mejia-Ramos, Weber \& Fuller, in press; Mason, Stylianides, \& Watson, 2013; Raman, 2003; Weber \& Alcock, 2009). In particular, diagrams may highlight properties of concepts that are not obvious from expressing these concepts symbolically; hence representing situations diagrammatically can suggest inferences that can be made and proving approaches that can be taken (e.g., Alcock \& Simpson, 2004; Gibson, 1998; Raman, 2003; Weber \& Alcock, 2004, 2009). However, students might not be able to reap such benefits, either 
because they are unaware of the roles that diagrams can play in proof writing (Raman, 2003) or they are not able to translate their visual insights into the language of proof (Zazkis, Weber \& Mejia-Ramos, 2014). Similarly, considering specific examples of general claims can provide insights that can be useful for writing a proof (e.g., Lockwood et al., 2013; Sandefur et al., 2013).

\subsection{Strategies and heuristics}

Expert mathematical problem solvers possess a large amount of strategic knowledge to help them solve problems. This includes heuristics of approaches that they can try when it is not obvious how to proceed, such as drawing a diagram and looking at simple cases (Polya, 1945; Schoenfeld, 1985) as well as "applicability conditions" that specify when a theorem or technique is likely to be useful when writing a proof (e.g., Anderson, Boyle, \& Yost, 1986; Weber, 2001).

\section{The Study}

\subsection{Goals of this study}

The goal of this study is to identify approaches that undergraduates successfully use to prove novel theorems in undergraduate mathematics. We do this with a finegrained analysis of six successful mathematics majors. The reason we chose to study successful mathematics majors is that we believed we were more likely to find productive behavior from students who were successful in their proof writing and in their advanced mathematics courses than by studying typical mathematics majors who are often unable to write proofs. Although we looked at successful students, the focus in this paper is on the strategies that they used rather than the students themselves. 
To avoid misinterpretation, we are not attempting to make what Firestone (1993) called a sample-to-population generalization, where we generalize attributes of our sample to some broader population. In particular, we are not claiming that the six successful mathematics students that we looked at are indicative of the broader population of all successful mathematics students. Likewise, we are not attempting to compare the behavior of these students with less successful students. Rather our focus is on what Firestone (1993) referred to as an analytic generalization where we attempt to generalize the qualitative results we found to a broader theory. In particular, we want to illustrate how particular proving approaches have the potential to help mathematics majors overcome or avoid impasses in the proving process.

\subsection{Participants}

This study was conducted at a large state university in the Northeastern United States with one of the top 25 mathematics departments in the country (US News and World report, 2014). We collected data over four semesters. After each semester, we sent e-mails to all students who had completed a second course in linear algebra inviting them to participate in our study. This second course in linear algebra was proof-oriented and had a transition-to-proof course as a prerequisite (as opposed to the first course in linear algebra which contained proofs but focused more on computation). The students in this course were almost exclusively mathematics majors. From this solicitation, 73 students participated in our study in exchange for payment.

\section{2. Instruments and protocol}

The design and aims of the study and protocol are similar to Schoenfeld (1985) in that we posed challenging novel proving tasks to our participants, but the content 
knowledge required to complete these proving tasks did not extend beyond a first course in calculus. Hence, we aimed to minimize the possibility that students' failures to write proofs were attributable to deficient content knowledge, allowing us to explore how students utilized their content knowledge when they worked on difficult proof construction tasks..

Participants were then given seven calculus proving tasks, one at a time, in a randomized order. These tasks are included in the Appendix. Participants were told to write a proof as if they were handing it in for a course examination. For each task, they were permitted to use a graphing calculation application on a computer while they completed the task. The application permitted them to perform basic arithmetic operations and graph functions with a high degree of resolution.

Participants were given ten to fifteen minutes to complete each task. They were allowed to stop working on a task at any time if they felt that they had obtained a written proof or they felt that they could make no more productive progress. After ten minutes had elapsed, the interviewer would suggest that the participant move on to the next task if the interviewer felt no productive progress was being made. After fifteen minutes, the work on the proof task was terminated, regardless of whether the interviewer and participant felt that productive progress was being made. Participants generally stopped making progress before this point was reached. This amount of time per task is similar to students' in-class exams, where they are typically asked to write four or five proofs in a 50 minute period. After a participant ceased working on a proof task, he or she was asked to describe the reasoning that they used for this task (retroactive recall). This process was repeated until the participant attempted all seven tasks. 


\section{3. Identifying highly successful students}

We used two measures to identify the most successful mathematics majors in our sample - from hereon, the highly successful students - performance on the proving tasks and performance in proof-oriented mathematics courses. For performance on proving tasks, one of two graduate student coders graded each of the written proofs produced by the participants using an adapted four point scale of Malone et al. (1980), which was used by Hart (1994) and Weber (2006) in their coding of students' proofs. A proof was scored as a 4 if it was a complete and valid proof, a 3 if it was a valid proof with the exception of minor and insignificant errors, a 2 if no proof was produced but substantial progress was made, and a 1 if no substantial progress was made. A proof was coded as correct if it received a score of a 3 or a 4 , incorrect otherwise. For purposes of inter-rater reliability, one fifth of the participants' proofs were randomly chosen to be evaluated by both graders. The graders agreed on the correctness of the proof $96 \%$ of the time (Cohen's kappa of .91) indicating a nearly perfect level of agreement.

Second, we obtained the participants' grades for three proof-oriented advanced mathematics courses - their transition-to-proof course, their second course in linear algebra, and their real analysis course. Completing the linear algebra course was a condition for participating in this study and completing the latter two courses are required for the mathematics major. Using the numerical scheme that the university where this study took place used to compute GPAs $(\mathrm{A}=4.0, \mathrm{~B}+=3.5 \mathrm{~B}=3.0, \mathrm{C}+=2.5$, and so on $)$, we computed their average grade across these three proof-oriented classes ${ }^{1}$. We note

\footnotetext{
${ }^{1}$ A small number of students were missing grades from their transition-to-proof course, most likely because they transferred credit for that course from another university. A small number of students were missing grades for their real analysis course because they completed an honors course in real analysis,
} 
there was a strong correlation between the number of proofs that a participant answered correctly and his or her mathematics GPA ( $r=.544)$, which is especially high given that the proving tasks were based in introductory calculus and thus did not assess the content knowledge required in these advanced courses. These proving tasks did not allow for the exploration of things like work ethic, the grading leniency of their course instructors, and so on. Hence, we infer that students' ability to prove novel theorems in a relatively short time span is predictive of their ability to succeed in advanced mathematics.

A student was designated as a highly successful student if, (i) they answered at least four of the seven proving tasks correctly, and (ii) their GPA in the three math courses we considered was a 4.0. Six participants met these criteria. Answering only four of seven tasks correctly might not seem like exceptional performance. However, two of the tasks in the study, tasks 5 and task 7, turned out to be highly difficult. Each of these was successfully answered by only one of the 73 participants in the study (both were amongst the six highly successful students in this study). We refer to these two tasks as the "extremely difficult tasks" and the other five tasks as the "ordinary tasks."

\begin{tabular}{|c|c|c|c|c|}
\hline \multirow[b]{2}{*}{$\begin{array}{l}\text { Participant } \\
\text { Group }\end{array}$} & \multicolumn{4}{|c|}{ Table 1. Aggregate performance of successful students and other participants } \\
\hline & $\begin{array}{l}\text { Perc } \\
\text { on o } \\
\text { (Tas }\end{array}$ & $\begin{array}{l}\text { tage correct } \\
\text { inary tasks } \\
1,2,3,4 \text {, and } 6)\end{array}$ & $\begin{array}{l}\text { Percentage correct } \\
\text { on exceptionally difficult } \\
\text { tasks (Tasks } 5 \text { and } 7 \text { ) }\end{array}$ & $\begin{array}{l}\text { Mean } \\
\text { math }\end{array}$ \\
\hline \multicolumn{2}{|l|}{$\begin{array}{l}\text { Successful } \\
\text { students }(\mathrm{N}=6)\end{array}$} & $83 \%$ & $17 \%$ & 4.00 \\
\hline \multicolumn{5}{|l|}{ Other } \\
\hline
\end{tabular}

Table 2. Successful students' performance by task

\begin{tabular}{llllllllr}
\hline Task & 1 & 2 & 3 & 4 & 5 & 6 & 7 & Total Correct \\
\hline
\end{tabular}

which fulfilled their real analysis requirement. In these cases, the participants' Math GPA was computed by averaging across the two grades that we had. 


\begin{tabular}{cllllllll}
\hline Successful stu \% Correct & $83 \%$ & $67 \%$ & $83 \%$ & $83 \%$ & $17 \%$ & $100 \%$ & $17 \%$ & $64 \%$ \\
Other students \% Correct & $12 \%$ & $7 \%$ & $49 \%$ & $39 \%$ & $0 \%$ & $43 \%$ & $0 \%$ & $22 \%$ \\
\hline Chase & $\sqrt{ }$ & $\sqrt{ }$ & $\sqrt{ }$ & $\sqrt{ }$ & & $\sqrt{ }$ & & 5 \\
Ronald & & $\sqrt{ }$ & $\sqrt{ }$ & $\sqrt{ }$ & & $\sqrt{ }$ & & 4 \\
Wolfe & $\sqrt{ }$ & & $\sqrt{ }$ & $\sqrt{ }$ & & $\sqrt{ }$ & $\sqrt{ }$ & 4 \\
Sarah & $\sqrt{ }$ & & & $\sqrt{ }$ & & $\sqrt{ }$ & $\sqrt{ }$ & 4 \\
Frank & $\sqrt{ }$ & $\sqrt{ }$ & $\sqrt{ }$ & & & $\sqrt{ }$ & & 4 \\
Theodore & $\sqrt{ }$ & $\sqrt{ }$ & $\sqrt{ }$ & $\sqrt{ }$ & $\sqrt{ }$ & $\sqrt{ }$ & & 6 \\
\hline
\end{tabular}

Table 1 documents which questions each highly successful student answered correctly. The combined success rate of all 73 participants on the ordinary tasks ranged from $12 \%$ to $52 \%$. A comparison of the highly successful students and the other 67 participants' math GPAs and their performance on the ordinary and extremely difficult tasks is presented in Table 1. The highly successful students' individual performance on each of the tasks is given in Table 2. As Table 1 documents, the highly successful students collectively answered $83 \%$ of the ordinary tasks correctly, whereas the remaining 67 participants in the study collectively answered only $30 \%$ of these tasks correctly. Table 1 also indicates that the other 67 participants had a collective math GPA of 3.15. Given the notorious difficulty of proof-oriented courses in university mathematics, having an average greater than a B indicates that these are above average students. Their disappointing performance on the proving tasks in this study serves as further evidence of mathematics majors' frequent inability to cope with proof. While there was little variance in performance amongst the participants on the extremely difficult tasks (almost everyone failed to produce a correct proof), they are worthwhile to include in our analysis as this can provide insight into what the highly successful students did when they reached an impasse.

\section{4. Analysis}

The analysis proceeded through four stages. In the first stage, the analysis was 
holistic and interpretive, where we explored participants' on-task proving behavior to get a general sense of how they approached the tasks. We also noted anything that we found to be distinctive or interesting about the participant's behavior. In the second stage, we continued analyzing participants' on task proving behavior, but now in a more systematic and targeted fashion. We specifically looked for how students chose a plan for how to proceed, how often they switched plans and the mathematical activities that surrounded these choices.

In the third stage of analysis, we examined participants' retroactive recall of their proof writing behavior. In watching this portion of the interview, we looked for evidence that either confirmed or contradicted our accounts of the first two stages of analysis. We also paid attention to reasoning that they cited that we did not observe during our first two passes through the data - when this occurred, we analyzed the proof construction phase of the data again to seek confirming evidence of the participants' comments from the retrospective recall portion of the interview. From these first three stages, we formulated hypotheses for how each individual participant approached the tasks and the competencies that they utilized to support these approaches. As an important caveat, we note that a failure of a participant to exhibit a competency does not imply that the participant lacked this competency. For instance, simply because a participant never drew a graph does not imply that this participant could not graph a function or draw inferences from it, only that this competency did not support this participant's proving behavior on these tasks.

The fourth stage of analysis involved member-checking interviews (Carlson, 2010). The purpose of conducting these member-checking interviews was to seek 
confirmatory evidence of our interpretations by sharing them with the participants and seeing if they thought we characterized them accurately. We invited the participants to meet with the first author for a member-checking interview. Five of the six highly successful students agreed to participate in these interviews; the remaining student, Frank, graduated from the university in question and could not be contacted. We structured the member-checking interviews as follows. For each of the categories we developed in the second stage of analysis we would ask a question. The questions were framed as, "We noticed that on these tasks, you usually did X [where X is some behavior or competency]. Do you think this is a fair interpretation of your behavior? Is this something that you usually do?" When appropriate, we showed a video clip of the participant engaging in behavior that we felt illustrated X. In nearly all cases, the participant agreed with our interpretation. Consequently, except for Frank who could not be reached for a member-checking interview, our conclusions are based on the triangulation of three data sources: participants' verbal/written work from their on task behavior, participants' immediate retrospective recalls of their proving processes, and the member-checking interviews.

\section{Results}

In our analysis, we distilled two qualitatively different strategies that the highly successful students used to generate proofs. We refer to these as the shotgun strategy and the targeted strategy. These two strategies are analogous to shotgun and targeted marketing strategies (e.g., Cahill, 1997).

A shotgun marketing strategy is characterized by raising awareness to a product to a large, non-specific group of people, such as placing fliers on cars in a high traffic area. 
This strategy was given its name because a shotgun fires a large number of smaller pellets in a wide area rather than aiming a single bullet at a specific target. To us the shotgun strategy for solving novel tasks is comprised of trying to prove a statement by quickly implementing a large number of plausible strategies in a short period of time. The term shotgun here is not intended to convey a random or haphazard plan choice; rather it is intended to convey that a prover is aware of general directions that might lead to a solution. One does not hit a target with the shotgun without shooting in its general direction.

The following characteristics were indicative of the shotgun strategy: (i) After reading the task statement, the prover expended little time or effort in understanding the statement to be proven. (ii) The prover did not spend much time in choosing or evaluating a plan to address the task. Rather, the prover usually implemented the first plan that came to mind without considering its likelihood of success. (iii) When implementing a plan, if the prover stopped making progress for a short time or perceived further progress to be difficult, helshe quickly either modified the plan or abandoned it. In the latter case, the prover quickly chose another plan and implemented that. In essence, immediately after reading the task the students who used the shotgun strategy engaged in a cycle in which they chose a plan, implemented a plan until it was not clear how to proceed, and then chose another plan. This process continued until a solution was obtained, they ran out of time to work on the task, or they ran out of plans to try. In this sense, impasses take up little of the students' time because they change approaches at the first sign that an impasse might be near.

In contrast to the shotgun strategy, some students applied a targeted strategy. In 
marketing, a targeted strategy is where one distributes tailored advertisements to a carefully chosen target group. In our context, we use targeted strategy to mean that the student carefully chooses a proving strategy based on a strong understanding of the theorem statement. The targeted strategy involved the following characteristics on the proving tasks: (i) When reading the task statement, the prover invested considerable effort in understanding what the statement was asserting. (ii) After representing the hypotheses of the task statement (often graphically), the prover would try to understand why the conclusion of the statement would be true. (iii) The proofs that resulted from the targeted strategy were often formalizations of intuitive (often graphical) arguments. (iv) Provers using the targeted strategy were reluctant to abandon the plan that they were implementing. They would only do so if they reached an impasse that they could not resolve for some time (usually several minutes). In this sense, the targeted strategy seeks to avoid reaching impasses by investing considerable time in choosing the right strategy that will not lead to an impasse.

We organized the results section as follows. In section 4.1, we illustrate the difference between shotgun and targeted strategies by showing representative responses to two tasks. In section 4.2, we discuss the shotgun strategy in more detail by presenting further illustrations of proof writing that use the shotgun strategy (4.2.1) and data from the member-checking interviews of students who used this strategy (4.2.2) In section 4.3, we do the same for the targeted strategy.

\section{1. Comparing shotgun and targeted strategies}

In this section, we contrast excerpts that illustrate shotgun and targeted strategies for solving the same task. Here we focused on episodes where participants were unable to 
successfully construct a proof. These allowed us to focus on how the two strategies

addressed impasses. We present successful proving episodes for the shotgun and targeted

strategies in 4.2.1 and 4.3.1, respectively.

\section{1. 1. Wolfe and Sarah's proofs on Task 2}

We begin by comparing the work of Wolfe and Sarah on task 2 (Prove that the

only real solution to the equation $x^{3}+5 x=3 x^{2}+\sin (x)$ is $x=0$ ). We begin with Wolfe's

work, which illustrates a shotgun strategy:

[1] [00:00] Prove that the only real solution to the equation $x^{3}+5 x=3 x^{2}+\sin (x)$ is $x=0$.

[2] [00:18] Well it's pretty obvious that zero is a solution, because if you just plug it in you get zero on both sides. Hurray.

[3] [00:29] So why is that the only real solution? Well let's see.

[4] [00:44] I'm just going to write down. I'll write down the thing, the equation but leave the $\sin (x)$ by itself.

[5] [00:50] So $\sin (x)=x^{3}+5 x-3 x^{2}$ which equals $x\left(x^{2}-3 x+5\right)$.

[6] [01:03] That doesn't factor nicely. And this is just tricky because of the $\sin (x)$ term.

[7] [01:13] So is there anything I can do here to make the problem easier?

[8] [01:20] Well squaring both sides wouldn't help.

[9] [01:28] Dividing by $\sin (x)$ wouldn't help.

[10] [01:32] Why is $\mathrm{x}$ the only real solution? Well usually with an $x^{3}$ term that means that you will have three solutions. Sometimes it means that they are complex.

[11] [01:49] So let me think of a way to prove those terms. Well I can't really think of any other x's that would work. Even complex. So I just have to work with what we got.

[12] [2:14] So zero obviously works. And then so if some sort of a polynomial I want that to equal $\sin (x)$ which is some periodic term. [sketching the graph of $\sin (x)$ ] That was a terrible $\sin (x)$ graph. But that's okay.

[13] [2:47] So $\sin (x)$ is periodic and how about I take the derivative of the part without the $\sin (x)$. So I get

$3 x^{2}-6 x^{2}+5$. Does that even factor? 15 could become 3 and 5 .. only 5 is prime so it's 1 and 5 . No, that doesn't really help. So taking the derivative doesn't really help.

[14] [03:18] So why would there be no other solutions other than the fact that $\mathrm{x}$ is trivially a solution?

[15] [03:30] Alright $\sin (x)$ just oscillates and I showed that the other stuff can factor into $\mathrm{x}$ and $\left(x^{2}-3 x+5\right)$.

Which well I know that that this only has a root at zero and this only has complex... or no, not complex roots.

[16] [04:12] So what can I use with $\sin$. Are there properties of $\sin (x)$ that can help with this? Well $\sin (x)$ is bounded between ... well $|\sin (\mathrm{x})| \leq 1$. So does putting a bound on it do anything? Well it does. Like I could try to make another equation that uses one or minus one and see if that goes anywhere I don't want to just be stuck on a problem. Um but it doesn't look like it would help either way.

[17] [04:59] So proving that the only solution is zero... what could I do.

[18] [05:10] Interviewer: So it's been about five minutes. Do you want to keep going on this one? Or go onto the next one?

[19] [05:13] [stares at the problem silently for $30 \mathrm{sec}$ ] I'm pretty sure I don't know what to do with this one so... we'll give up on this one.

We observed several aspects of this transcript that are representative of the shotgun strategy. First, note that little effort was made trying to understand the task. 
Immediately after reading the task in [1], Wolfe began doing calculations in [2] to establish the trivial part of the task statement, that $\mathrm{x}=0$ is a solution. After that, Wolfe spent only 15 seconds reflecting before attempting to factor the expression in [4-5]. He began his attempts at proving the statement with little time spent understanding the statement or formulating a plan. Second, Wolfe proposed numerous plans, such as factoring the polynomial [4], squaring both sides [8], dividing by sine [9], showing that all but one of the roots to the cubic part of the equation are complex [10], and using the fact that sine is bounded [16]. Most of these plans were dismissed quickly, although we also acknowledge that they do not seem promising. Third, Wolfe asked "why" the statement would be true at three points ([3], [10], [14]), but he did not appear to seek an intuitive explanation for why the proven statement is true; rather he responded by proposing techniques that he could plausibly use to establish the theorem. Finally, Wolfe generated many plausible plans by thinking about the mathematical concepts in the statement to be proven and listed proving techniques and facts about these concepts. These include ideas about solving equations (e.g., factoring polynomials, squaring both sides), cubic equations (considering complex roots), and the sine function (the function is bounded between -1 and 1). Generating ideas in this way was not useful to Wolfe for this task, but as we show in 4.2.1, such idea generation often provided students implementing the shotgun strategy with insights that led to clever proofs. We compare Wolfe's proof attempt with the work of Sarah, which illustrates a targeted strategy on the same task.

[1] [00:00] Prove that the only real solution to the equation $x^{3}+5 x=3 x^{2}+\sin (x)$ is $x=0$. Oh great [sarcastic]

[2] [00:15] Interviewer: What are you thinking about?

[3] [00:16] How do I do this? I don't like this. So we have $x^{3}-3 x^{2}+5 x=\sin (x)$. So what does this function look like? Hey I might actually use the calculator thing.

[4] [00:52] Interviewer: Alright. Yeah here. You want to graph a new function?

[5] [01:10] [Sarah and the interviewer work together to use the graphing software to plot both the graph of the polynomial part of the equation, $f(x)=x^{3}+5 x-3 x^{2}$ and $\left.\sin (x)\right]$ 
[6] [01:58] So what is there to show here? Both functions are increasing there but one is increasing faster than the other. So they intersect right there and that function is increasing faster.

[7] [02:43] Alright. So if you take the derivative $3 x^{2}-6 x+5$ and then the derivative of $\sin (x)$ is $-\cos (x)$. So what does this mean at $x=0$ ? At zero it's going to be 5 and the derivative of this thing is going to be between zero and one. So and after this this function is going to continue increase. Why does it continue to increase? Alright, so we have $3 x^{2}-6 x+5$. Um.... So right now I'm trying to show why it never intersects again after $\mathrm{x}$ is equal to zero. And I think that's because it intersects once and then it can't intersect again because the polynomial increases so much faster than the $\sin (x)$ function. I'm a bit confused as to why it continues to increase afterwards though. I mean this is given by $\mathrm{f}^{\text {' }}(\mathrm{x})$. And this is always going to.. well this is $3 x$. When is this thing going to be equal to zero?

[8] [04:53] $6 x+5$. Ohhh $-b \pm \sqrt{b^{2}-4 a c . ~} 3$ times 5 so that's going to be 15 times something so this is going to be negative. So this is never going to be equal to zero. Which explains why the function is always increasing. I guess. Instead of switching around. Okay so.

[9] [05:39][Sarah begins writing a proof] $x^{3}+5 x=3 x^{2}+\sin (x)$ is the same problem as $x^{3}-3 x^{2}+5 x=\sin (x)$. Clearly $x=0$ is a solution because we can just plug it in. Solution. Um lets call this thing $f(x)$. So $f^{\prime}(x)$ is actually always increasing. Right? Yeah. Can I clear this and graph something instead? [Sarah graphs the derivative, $\left.f^{\prime}(x)=3 x^{2}+5-6 x\right]$

[10] [06:45] Thanks, $3 x$, oh, $3 x^{2}+5$, hold on. $3 x^{2}+5-6 x$. That's hopefully the derivative of this thing. It's starts decreasing after that. $3 x^{2}-6 x+5$. Oh wait no it doesn't have any $\mathrm{x}$ intercepts. But...

[11] [07:51] Anyway that's the original function oh yeah here it... looks like it's negative. Oh this function is always increasing. [Sarah works unsuccessfully to show that $f(x)=x^{3}+5 x-3 x^{2}$ and $\sin (x)$ only intersect at zero by showing that $f^{\prime}(x)>\cos (x)$. She deals with $\mathrm{x}>0$ and $\mathrm{x}<0$ as separate cases. She ends up running out of time before she is able to complete a rigorous proof.]

There are several aspects of this transcript worth noting that are representative of the targeted strategy. First, Sarah did not begin doing calculations until 2:43 had elapsed when she computed the derivative of $f(x)$ in [7]. Prior to that point, Sarah had been working to represent the task that she was asked to solve to see why its statement might be true. We can compare this with Wolfe, who like other students implementing the shotgun strategy began doing calculations almost immediately after reading the task.

Second, Sarah developed graphical arguments for why the task statement was true, which she then set as a goal to formalize. In [6], she noted that $f(x)$ is increasing faster than $\sin (x)$, which led to her taking the derivative in [7]. The calculation that she undertook was not aimless or exploratory, but based on how she understood the task situation, which again can be contrasted with some of Wolfe's ideas, such as squaring both sides of the equation or dividing the equation by sine.

In Figure 1, we adapt Schoenfeld's (1987) metacognition plots to compare how 
Wolfe and Sarah spent their time trying to write this proof. In the figure shotgun and targeted strategies are indicated with $(\mathrm{S})$ and $(\mathrm{T})$, respectively. As can be seen, Sarah spent substantially more time than Wolfe in trying to understand the task statement and formulate a plan. Wolfe suggested multiple plans in quick succession, while Sarah adopted only a single plan.

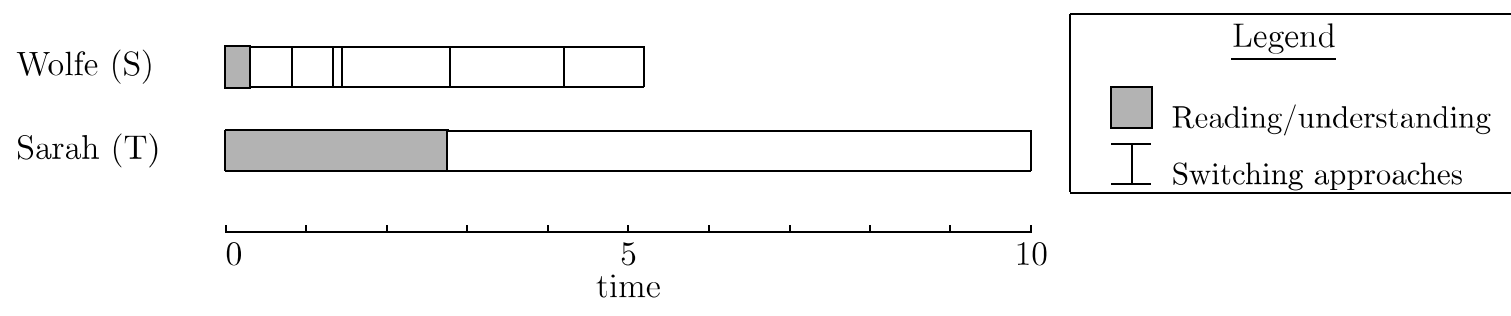

Figure 1: Sarah and Wolfe work on task 2

\section{1. 2. Chase and Theodore's work on Task 7}

Task 7 stated, "Let $f$ be differentiable on $[0,1]$, and suppose that $f(0)=0$ and $f^{\prime}$ is increasing on $[0,1]$. Prove that $g(x)=\frac{f(x)}{x}$ is increasing on $(0,1)$ ". Chase's transcript is given below and illustrates a shotgun strategy:

[1] [00:00] Okay, lets see. Differentiable at zero, increasing and that's increasing.

[2] [00:11] Lets see.

[3] [00:14] I'll just take the derivative. Which is [writes $g^{\prime}(x)=\frac{f^{\prime}(x)-f(x)}{x^{2}}>0$ ].

[4] [00:41] Let's see if this is positive, it is positive. Um... Well that is differentiable and...

[5] [01:06] Interviewer: What are you thinking about?

[6] [01:07] Um I'm just trying to see if I can make this $\left[g^{\prime}(x)\right]$ positive. So f prime is increasing doesn't necessarily mean that this is positive. What if this is increasing and that is increasing and this could be. [writes $f^{\prime}(x)>0$ ] So if it's smaller than that then.. no [crosses out $f^{\prime}(x)>0$ ].

[7] [01:54] So that is... Okay, so that's a difference quotient, which means that it's always. It always equals to some.. something there [writes: $g(x)=\frac{f(x)-f(0)}{x-0}=f^{\prime}(c)$ ] Um by mean value theorem [writes: MVT with arrow toward $\left.\mathrm{f}^{(\mathrm{c})}\right]$. But does that have to be positive? Um.. not necessarily.

[8] [02:46] Definitely, I'm thinking really slow. I'm thinking about food. Lets see. That much is certain. and $\mathrm{f}$ prime is increasing but we are not.

[9] [03:07] Oh wait wait wait. Oh yeah yeah yeah. That makes sense. Fix zero x y. Um... one [writes $0<x<y<1]$.. We observe that first of all. Uh that's not going to get us anywhere. So. We observe that $\mathrm{g} \mathrm{x}$ minus $\mathrm{g}$ equals $\mathrm{f}$ oh $\mathrm{I}$ want to divide $\mathrm{x}$ fy by $\mathrm{fx}$ which is [writing quietly

$\left.g(y)-g(x)=\frac{f(y)}{y}-\frac{f(x)}{x}=\frac{f(y-0)}{y-0}-\frac{f(x-0)}{x-0}=f^{\prime}\left(c_{1}\right)-f^{\prime}\left(c_{2}\right)\right]$. For some.. $\left[\mathrm{c}_{1}\right.$ in $(0, \mathrm{x})$ and $\mathrm{c}_{2}$ in $\left.(0, \mathrm{y})\right]$.

The next equality follows from the mean value theorem. 
[10] [04:59] Can I guarantee that $\mathrm{C}_{2}$ is greater than $\mathrm{C}_{1}$ ? Since $\mathrm{f}$ prime is increasing we have $\mathrm{f} \mathrm{c} 1$ greater than $\mathrm{f} \mathrm{c}$ 2. So yeah that's true. [writes: since $\mathrm{f}^{\prime \prime}$ is increasing we have $g(y)>g(x)$ if $c_{1}<c_{2}$ ].

[11] [06:24] Interviewer: What are you thinking about?

[12] [06:32] I'm thinking about modifying this slightly. [writes on scratch paper

$\left.\frac{f(y)-f(y-x)}{y-(y-x)}-\frac{f(x)-f(0)}{x-0}\right]$ Um. no no no. [Scratches out previous work]. Man I really can't think.

[From this point forward, Chase makes no further progress on the task before giving up at 8:06].

We highlight some features of this excerpt that are consistent with the shotgun strategy. First, notice that Chase began formulating a plan [2] eleven seconds after reading the task [1], putting little effort into initially developing an understanding of the task statement. He formulated his first plan, taking the derivative in [3], three seconds later without any evidence that he considered if this plan was likely to be successful.

Nonetheless, Chase gave up on this plan quickly. In [7], Chase tried to use the Mean Value Theorem, which capitalized on the fact that $f(0)=0$, but since the value of $f^{\prime}(x)$ could be positive or negative, he rejected this plan as not providing enough information. (There is an error in the equation Chase wrote here. The $f^{\prime}(x)$ should be a $c$ where $0<c<x$ ). In [9], Chase tried an algebraic plan, which tried to show $g(y)-g(x)>0$ by fixing $y$ and $x$, but was initially unsuccessful. In [12] Chase set up a different algebraic equation (using $y$ and $y$ - $x$ instead of $x$ and $y$ ), but made no progress and gave up.

Note that for each of the four plans that Chase implemented, he spent under two minutes on each and he abandoned the plan without showing evidence of considering why the plan would be unsuccessful. We compare Chase's work with Theodore's proof attempt, which illustrates a targeted strategy on the same task:

[1] [00:00] $f(x)$ differentiable on zero one. zero one. Suppose that $f(0)=0$ and $f^{\prime}(x)$ is increasing, that is $f^{\prime \prime}(0)>0 . f^{\prime}(x)$ is increasing. Well actually the definition of increasing means it could remain flat so $f^{\prime \prime}(0) \geq 0$. Um okay so we have some sort of slope that is increasing. Prove $g(x)=f(x) / x$ is increasing on zero one. [2] [01:31] That is $g^{\prime \prime}(x) \geq 0$. Okay, so $g^{\prime}(x)$. I mean I have $g(x)$ so I can just figure out what $g$ " $(x)$ is. So I'll use the, whatever the division, $\mathrm{f}$ prime of $\mathrm{x}, \mathrm{x}$ minus $\mathrm{f}$ of $\mathrm{x}$. $\mathrm{x}$ squared. This is going to be annoying. here we write this $\left(f^{\prime}(x) x-f(x)\right) / x^{2}$. Here I'll have $f^{\prime \prime}(x) . \mathrm{x}^{2} .-\mathrm{f}(\mathrm{x}) / \mathrm{x}^{2}$ squared. 2x. All over [recalculating second derivative silently] I'm just rewriting it. I don't know if that's actually going to be useful or not. I want to show that this $[\mathrm{g},(\mathrm{x})]$ is positive. 
[3] [03:32] I know that's positive or non-negative $\mathrm{x}$ is non-negative so this term is non-negative I know $f^{\prime}(x)$ prime is increasing but I don't know what it is. Um. So I can't really say that this is greater than zero. I cannot.

[4] [04:27] Well if $f^{\prime}(x)$ is increasing then $f(x)$ is increasing... I'm trying to think of an elegant way to do this. It has something to do with the fact that. Like if I just had $f(x)$ increasing then when I divide by $\mathrm{x}$ which is positive that would kind of flatten $f(x)$. But since I have f prime increasing it's enough to divide by $f(x)$ and still have an increasing function. I want to use L'Hopital's rule because I see a correlation. [5] [05:35] Wait I want to show that $g(x)$ is increasing that means I want to show that $g^{\prime}(x) \geq 0$. Not $g^{\prime \prime}(x)$. So I made a mistake. That was a waste of time.

[6] [06:34] Well $x$ is greater than or equal to zero so we just need $\left(f^{\prime}(x) x-f(x)\right) \geq 0$. That is, we need $f^{\prime}(x) x \geq f(x)$.

[7] [07:17] Let me use what I know. $\mathrm{f}$ " $(\mathrm{x}) \geq 0$ and both sides. And $f^{\prime}(x)$ is ... is $f^{\prime}(x)$ increasing. $f^{\prime}(x)$ is increasing. So it's greater than or equal to some constant but just having it greater than a constant doesn't increase it.

[8] [08:21] Interviewer: What are you thinking about?

[9] [08:22] I'm thinking that this should imply that $f^{\prime}(x)$ is increasing but I used it and all I got was that it's above a line, that it's greater that a constant. I should be getting more out of that.

We highlight features consistent with the targeted strategy. First, notice that Theodore spent substantial time (1:31) simply reading the question. Second Theodore's initial strategy emerged from attempts to understand the task [2]. In formulating his understanding of the statement, he (incorrectly) deduced that he wanted to show that $g^{\prime \prime}(x)>0$. Third, once Theodore chose a strategy, he spent over four minutes attempting to implement the strategy (from [2-5]). Indeed, the reason that Theodore switched strategies is because he realized he had made a mistake earlier in the task. Finally, in [4], we see Theodore's attempt to build an intuitive argument for why $g$ ' $(x)>0$. He was unsuccessful, but as we show in 4.3.1, when students using the targeted strategy do construct such arguments, they try to write their proof by formalizing the intuitive ideas in the argument. For now, we note that Theodore tried in [4] to take "an elegant approach", which can be contrasted with Wolfe, who (as we show in 4.2.2 below) referred to his method of proving as taking a "brute force" approach.

In Figure 2, we present Chase and Theodore's activities in their proof attempts as a function of time. Like Wolfe, notice that Chase spent little time reading the task or formulating a plan, but implemented multiple plans during his proof attempt. In contrast, 
Theodore spent more time reading the task and was more persistent with the plan that he chose to implement.
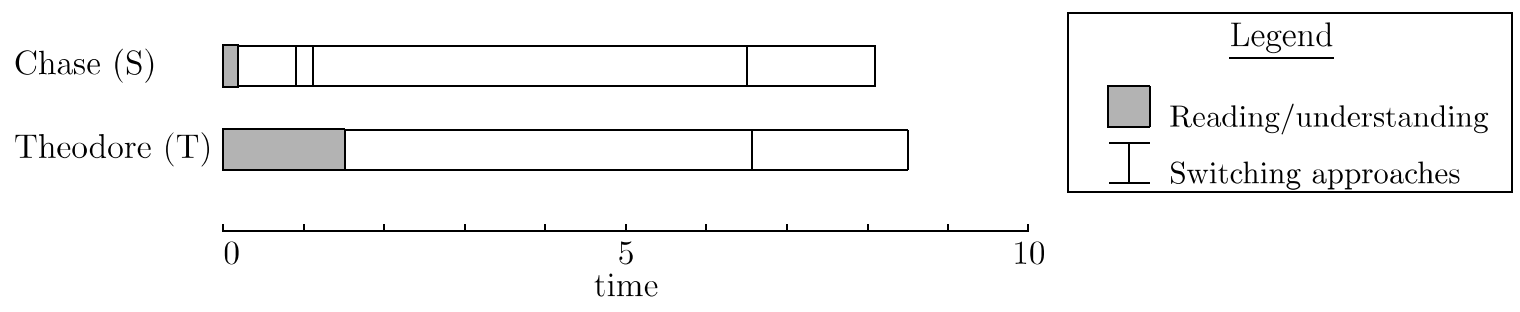

Figure 2: Theodore and Chase work on task 7

\section{1. 3. Summary of differences targeted and shotgun strategies}

The excerpts above illustrate that the shotgun strategy involves immediately diving into a task, implementing a plan with little time spent understanding the task and switching plans frequently. In contrast, implementing the targeted strategy involves substantial time spent trying to understand the task. Subsequent plans are based on this understanding.

\section{2. The Shotgun Strategy}

\section{2. 1. Excerpts of Successful Implementations of the Shotgun Strategy}

In section 4.1 we focused on episodes where the highly successful students were unable to produce proofs. This highlighted what the highly successful students did when they reached impasses and illustrated the differences between shotgun and targeted strategies. However, this focus on unsuccessful proofs may have given the reader a false impression regarding the mathematical abilities of the highly successful students. In addition to showing that the highly successful students often succeed on proof writing tasks, this analysis allows the reader to see how shotgun and targeted strategies could yield valid proofs.

We begin by discussing an excerpt of Ronald working on task 4 (Prove that 
$\mathrm{A}^{2}+\mathrm{AB}+\mathrm{B}^{2} \geq 0$ for all real numbers $\mathrm{A}$ and $\left.\mathrm{B}\right)$.

[1] [00:00] Alright. Prove that $\mathrm{A}^{2}+\mathrm{AB}+\mathrm{B}^{2} \geq 0$ for all real numbers $\mathrm{A}$ and $\mathrm{B}$.

[2] [00:07] Alright well um... hmmm is greater than or equal to zero.

[3] [00:13] Well this is a sum of three terms so one thing we could use is maybe the arithmetic mean geometric mean thing.

[4] [00:24] Lets see if that works out. Well we have let see, what does the arithmetic mean thing say. $(x+y+z) / 3 \geq 3 \sqrt{x y z}$. And if we can show that maybe ${ }^{3} \sqrt{x y z} \geq 0$ then we are done.

[5] [00:42] So lets see $A^{2}+A B+B^{2}$ is equal to, not equal to, greater than or equal to, three times the cube root of their product. So that would be oh wow $A^{3} B^{3}$, [writes $\left.\sqrt{A^{2} A B B^{2}}=\sqrt{A^{3} B^{3}}=A B\right]$, which is equal to [writes $\left.\left(\mathrm{A}^{2}+\mathrm{AB}+\mathrm{B}^{2}\right) / 3\right]$.

[6] [00:59] Wait, wait wait a second. Um....oh. The cubed root of A cubed B. That's 3AB. hmmm.

$\left[3 \mathrm{AB} \leq \mathrm{A}^{2}+\mathrm{AB}+\mathrm{B}^{2}\right]$. Alright well this is not necessarily equal to zero for all $\mathrm{A}$ and $\mathrm{B}$ so.

[7] [01:09] I guess that doesn't really work.

[8] [01:20] Or maybe I'm doing this wrong. Anyways well.

[9] [01:22] The other thing I noticed was that this term appears in the factorization of $\mathrm{A}^{3}-\mathrm{B}^{3}$.

$[10][01: 36]$ So $A^{3}-B^{3}=(A-B)\left(A^{2}+A B+B^{2}\right)$. Alright lets see.

[11] [01:49] Alright well if we assume that $A \geq B$ then.... we should be able to..

[12] [02:08] Alright. So I guess since this term $A^{2}+A B+B^{2}$ is symmetric. So I guess I'll write this down here too. Because of the symmetry of, I don't even know how to write this, of the above term. We can assume without loss of generality, which I will abbreviate here as WLOG. That $\mathrm{A} \geq \mathrm{B}$. Then $\mathrm{A}-\mathrm{B}$ is greater than or equal to zero. So, So $A-B \geq 0$ and $A^{3}-B^{3} \geq 0$. And well that implies that well that. Well lets see, we'll put this on a separate line, but, $A^{3}-B^{3} \geq 0$ implies that it's factorization $(A-B)\left(A^{2}+A B+B^{2}\right) \geq 0$. And I guess I'll have to be a little careful here. We'll split this into two cases.

[Ronald proceeds to write a proof with two cases. Case 1: $A=B \cdot A^{2}+A B+B^{2}=A^{2}+A^{2}+A^{2}=3 A^{2} \geq 0$. Case 2: $A>B=>B$ oth $A^{3}-B^{3}=A-B$ are positive. Since $A^{3}-B^{3}=(A-B)\left(A^{2}+A B+B^{2}\right), A^{2}+A B+B^{2}$ is also positive. The entire proof takes $4: 37]$

In the above, after reading the task in [1], Ronald spent only six seconds reflecting on the task before choosing his first strategy in [3], the arithmetic mean geometric mean inequality. Much like the other shotgun strategy excerpts, Ronald spent little time reflecting on the task before choosing an initial strategy. Next notice that Ronald abandoned the arithmetic mean/geometric mean strategy after encountering difficulty in [9]. This happened about one minute after choosing the strategy. Although he expressed that his difficulty may have been due to a calculation error in [8], he chose to immediately switch to a new strategy in [9], using the fact that $\mathrm{A}^{2}+\mathrm{AB}+\mathrm{B}^{2}$ appears in the factorization of $\mathrm{A}^{3}-\mathrm{B}^{3}$. This strategy resulted in a clever solution, which Ronald proceeded to write up.

Next we discuss an excerpt of Chase's work on task 2 (Prove that the only real 
solution to the equation $x^{3}+5 x=3 x^{2}+\sin (x)$ is $\left.x=0\right)$ :

[1] [00:00] Prove that the only real solution to that is $\mathrm{x}$ equals zero.

[2] [00:04] Okay, only real solution to that equation interesting.

[3] [00:12] So since I can't actually compute that most likely I have to use intermediate value theorem.

[4] [00:36] And let's see this function [writes $\left.f(x)=x^{3}+5 x-3 x^{2}-\sin (x)\right]$ are you increasing strictly? My gut feeling says no. But I'll compute the derivative any way, [writes: $\left.f^{\prime}(x)=3 x^{2}+5-6 x-\cos (x)\right]$.

[5] [01:08] This is bounded below by -1 because that's the max [writes $\left.\geq 3 x^{\wedge} 2+5-6 x-1\right]$ and deal with that, that is four $\left[=3 x^{2}-6 x+4\right]$. Do you ever go down below? Let's see. $x^{2}, 2 x$, oh that's an ugly thing [writes $3\left(x^{2}-\right.$

$2 x+4 / 3)$ ]. That would be $b^{2}-4 a c$ [writes: $\left.2 \pm \sqrt{ } 4-\right](4)(1)(4 / 3)$ that is definitely bigger than 4 [writes $16 / 3>4$ ]

which means that all the roots are imaginary. Which means that it doesn't touch that $\mathrm{x}$-axis, which means

that this is always strictly bigger than zero. Which means this $[f(x)]$ is strictly increasing so there is only

one real solution if it passes the $\mathrm{x}$-axis.

[Chase proceeds to write up the above as a formal proof]

As with the previous shotgun strategy excerpts, Chase spent little time trying to

understand the task. Eight seconds after reading the task he reflected that the intermediate value theorem could be useful in [3]. Immediately after this he settled on showing that the function as only one solution by showing that it is strictly increasing [4]. He pursued this strategy in spite of expressing a "gut feeling" that it would not work. This illustrates that students implementing a shotgun strategy may not have a high degree of confidence in the strategies they try. This provides illustrative evidence that Chase was more interested in trying out plausible approaches to solving the task rather than carefully selecting an approach that was guaranteed to work. This contrasts with students implementing the targeted strategy. Those students commonly based their proofs on informal understandings of tasks and hence tended to pursue strategies they believed would work.

\section{2. 2. Member-checking interviews}

In their member-checking interviews, each student who was identified as using the shotgun strategy in this study was asked, "I noticed that as soon as you would try to solve the task, you'd dive right in. Some students spent a lot of time trying to understand the statement and carefully chose a plan of attack. But you started trying things right 
away. Do you think that's a fair assessment?" All three students confirmed our

assessment:

Wolfe: Yeah. Like when a lot of my friends see me do problems, they always comment on how appears I'm just brute forcing the problem in the very beginning. And the way I usually tell them is like you know brute force is usually just going to solve the problem. The problem is how long it would take? (italics are our emphasis).

Chase: I think it's fair and unfair because, you guys, the sample was undergrads. Interviewer: Right it was undergrads. Chase: And when that took place, which is when I was maybe junior or senior there were very few undergrads who were serious math students at the time. So I'm an unfair outlier in that sample in that case.

Ronald: Yeah.

When asked if this was something that they ordinarily did, each student also agreed with our descriptions. In Chase's excerpt, he acknowledged that he was described accurately, but pointed out that this study occurred with undergraduates. We discuss this distinction with further excerpts from Chase shortly. In their member-checking interviews, each of the three students who primarily employed the shotgun strategy was also asked, "I noticed that when you started these problems, you would think of approaches that you could try, try each one and see how far that got you, and then chose a new approach and see how far that got you, and do that until you solved the problem. Do you think that's a fair assessment?" The students' responses are given below:

Ronald: Definitely. Yeah. It's sort of um. I do like to throw out as many ideas as I can. And yeah that sounds about right. (Italics are our emphasis)

Chase: Well, yes, but I hesitate to say because isn't that what everyone does?

Wolfe: Right. That sounds exactly like me.

Later in the member-checking interview, Chase provided some insight into why he chose to use the shotgun strategy on the tasks in this study. At the time of the interview, Chase was a doctoral student in mathematics at a university with a prestigious mathematics department. Chase claimed his shotgun strategy was appropriate because all of the statements he was asked to prove should have had an easy solution. This belief was 
based on Chase's confidence in his abilities, where he considered himself as an "outlier" since unlike most of his undergraduate peers, he was a "serious math student".

Chase: When [the study] took place, which is when I was a junior or a senior, there were very few undergraduates who were serious math students at the time. So I'm an unfair outlier.

Since he was a strong student, he believed problems in calculus (and in much of the undergraduate curriculum) should be "trivial" to him.

Chase: The way mathematical training works, as far as I understand it. If you get a good enough education, after a certain point, everything before becomes trivial [...] Most of those basic problems should be trivial to me. If I can't solve it immediately, my training is such that I should be able to tell you immediately this is probably how you do it.

Thus when Chase was working on the proving tasks in our study, he claimed, "I sort of went in with the assumption that if I can't do it immediately, it probably means I'm doing it wrong". This is similar to an excerpt from Wolfe earlier, where he said that "brute force" was capable of solving most of the tasks that students would encounter. It is interesting to note that Chase indicated that he was trying to refrain from using the shotgun strategy in graduate school.

Chase: I'm changing now. I'm less likely to do that now that I'm in grad school and I'm learning the values of dirty computations. [...] Are you aware of the soft analysis versus hard analysis distinction?

Interviewer: No.

Chase: So in the field of mathematical analysis, there is this colloquial distinction called soft analysis and hard analysis. And a very elementary example of hard analysis would be an epsilon-delta proof. Where you actually have to do it. Where as soft analysis would say something like, by this topological theorem this works. And I was very much a soft analysis person. So if there is more than five lines of hard analysis I was like "okay, I don't really want to do this".

Interviewer: Okay, so you would look for 'is there a theorem I could do this quick and dirty with?' Chase: Right.

The notion of Chase doing "soft analysis" was consistent with our analysis of his behavior on the proof construction tasks. When Chase abandoned a plan, it was often not because the plan was guaranteed to fail, but because Chase would have to do substantial work to make it succeed. Chase provided a reason why this strategy might not be sufficient in his area of research. 
Chase: So one thing is that it's sort of like a self-discipline thing now that I'm pursuing a professional career in mathematics. I'm just telling myself okay, if I continue to be lazy I'm probably not going to make it so I should probably care about this a little more. And that's one big reason. Another is the fields that I'm interested in. Harmonic analysis. Differential analysis. It just happens that a lot of what I'm interested in, most of the easy stuff has been done already. I sort of have to do hard analysis as well.

Interviewer: Okay so if there's like if there is an easy theorem you could use the result has probably been published already. So now you are sort of shifting away from...

Chase: Right. But I will admit that if I could make a career just doing soft analysis I probably still would.

Here Chase drew an interesting distinction between the statements he was asked to prove in his undergraduate education (including those in our study) and the open questions in his area of research. Most of the statements in our study lent themselves to the easy application of theorems that Chase was aware of, so Chase viewed his job as locating the right theorem. He is forced to adjust his proving strategies in his doctoral program since the "easy theorems" that can be addressed in that way have all been proven. This excerpt provides evidence that preference for shotgun or targeted strategies may be task/situation dependent and not a static personal preference.

\subsubsection{Dealing with impasses}

Most problem-solving models available in the literature emphasize understanding a problem before implementing a plan to solve it (Carlson \& Bloom, 2005; Polya, 1957; Schoenfeld, 1985). The shotgun strategy excerpts are not consistent with this; the students began implementing a plan very early in the proof construction process. Further, as we illustrated with Chase in 4.2.1, students implementing a shotgun strategy sometimes chose a plan even if they did not believe the plan was likely to be successful. Schoenfeld (1985) observed that the typical problem solver who did this would sometimes find themselves "chasing wild mathematical geese"-- that is, pursuing a plan that was doomed from the start. It is interesting to observe how a shotgun strategy enables a student to avoid chasing mathematical geese. They did so by switching plans frequently. They often switched plans the first time progress implementing a plan ceased 
to progress smoothly.

\section{3. Targeted Strategy}

\section{3. 1. Excerpts of successful implementations of the targeted strategy}

In this section we explore successful proof attempts that utilized the targeted strategy. We begin by discussing the work of Frank on task 1 (Suppose $f(0)=f^{\prime}(0)=1$. Suppose $f^{\prime \prime}(x)>0$ for all positive x. Prove that $\left.f(2)>2\right)$.

[1] [00:00] Suppose $f(0)=f^{\prime}(0)=1$. Suppose $f^{\prime \prime}(x)>0$ for all positive. Prove that $f(2)>2$.

[2] [00:11] Okay. So $f(0)=f^{\prime}(0)=1$. Okay, so if $f^{\prime \prime}(x)>0$ then the function is concave upward. So $f(x)$ would be like that [sketches concave up line]. Something like that, for all positive $\mathrm{x}$.

[3] [00:35] [sketching graph as he talks] So $f(0)=1$. So one is a root of $f(x)$. So if we have one $f . . . f(0)$ has a root at one and it's concave up for all positive x. So it would be something like that. And $f^{\prime}(0)=1$, which means that it's increasing after one. At one it has a slope of one. No at zero it has a slope of one. Okay $f(0)$. This was not the right drawing at zero. At zero $f(0)=l$ and for all positive $\mathrm{x}$ we have $f^{\prime \prime}(x)>0$, so it's concave up here, from zero onward. And $f^{\prime}(0)=1$, which means that we have a slope of one at zero.

[4] [02:08] Now we need $f(2)>2$ is greater than two. So how do we get $f(2)$ ?

[5] [02:19] Well okay because it's concave up it's increasing and so $f(2)$ is certainly greater than one. because $f(0)=1$. So two is somewhere up here. $f(0)=1 . f(2)$ has to be greater than one. So the derivative, that should help. At zero it has a slope of one. But then that changes and moves us away from zero. $f(2)$ has to be greater than one. I need it to be greater than two. So if $f(2)=2$ then we have at one, zero we have one. And at two we have two. So this is we have two... this is still concave up and our slope at $(0,1)$, that means a line $f(x)=1 \ldots$ is so that... it's okay. So $y=x$ plus something is tangent to this guy $[f(x)]$ at zero. So slope of tangent at zero is one. Okay so tangent line $L(x)=x+b$. And we know that it intersects at zero and $f(0)=1$. So this also has to have this is at zero. It intersects $f(0)$ at one... So it intersects here. So this also has $(0,1)$ as a point. So one equals $b$ so $b=1$. So the tangent line equals $x+1$ at zero.

[6] [6:03] Tangent line is $L(x)=x+1$. This is $f(x)$ this is ... since the tangent line is $L(x)=x+1$ and we have that this is concave up that means that after one $f(x)$ has to be greater than $x+1$. So $f(x)>x+1$. So $f(2)>2$.

[7] [6:31] Final proof. [Frank proceeds to write up his argument in [6] into a formal proof]

After reading the task in [1] Frank spent time recalling the limit definition of derivative in [2] and constructed a graph of a generic even function in [3]. This took over a minute.

Spending significant amounts of time coming to grips with a task was typical of implementations of the targeted strategy. The graph Frank produced while working to understand the task was then used to construct an informal argument regarding why the result held [4]. The translation of this argument was the basis of his proof strategy. Frank worked to formalize his intuitive argument by relating it to notation in [5] and then used this notation to write a rigorous proof. 
Now we explore another excerpt that illustrates successful implementation of a targeted strategy, the work of Theodore on task 3 (Suppose $\mathrm{f}(\mathrm{x})$ is a differentiable even function. Prove that $f^{\prime}(x)$ is an odd function.):

[1] [00:00] Okay, suppose $f(x)$ is differentiable and even. Prove that $\mathrm{f}$ prime is odd.

[2] [00:12] Alright, so $f(x)$ is differentiable so it's like continuous and smooth. What's the definition? I need the definition of differentiability, which is an annoying one, $\lim _{h \rightarrow 0}(f(x)-f(x+h)) / h$ exists as $\mathrm{h} \rightarrow 0$ from the left or right.

[3] [00:43] $f(x)$ is even. So let me just draw what an even function might look like. So across the origin. [Draws an arbitrary function with reflectional symmetry about the y-axis].

[4] [01:16] So basically, if I look at a slope on this function [draws a tangent on the negative side of the previously drawn function], then I look on the other side [draws a mirror tangent on the positive side], it's the same slope but negative. So that's going to show that $\mathrm{f}$ prime is odd.

[5] [01:41] So if I look at the definition of derivative as a slope and then I find the derivative on the negative side using the fact that it's even I should get the negative of the derivative, showing that $f$ prime is odd.

[6] [01:56] [Theodore writes up a formal proof showing that- $f^{\prime}(x)=-f^{\prime}(-x)$ by manipulating the limit definition of $f^{\prime}(-x)$ until it looks like $-f^{\prime}(x)$.]

After reading the task in [1] Theodore spent time recalling the limit definition of derivative in [2] and constructed a graph of a generic even function in [3]. This took over a minute. Spending significant amounts of time getting to grips with a task was typical of the work of students implementing the targeted strategy. The graph Theodore produced while working to understand the task was then used to construct an informal argument regarding why the result held [4]. The translation of this argument was the basis of his proof strategy. Theodore worked to formalize his intuitive argument by relating it to notation in [5] and then used this notation to write a rigorous proof.

The excerpts in this section helped illustrate that successful implementation of the targeted strategy involves a lengthy reading and exploration phase that often culminates in the creation of an informal argument. These informal arguments form the basis of the resulting proofs. The targeted strategy is often more time consuming than the shotgun strategy. But the resulting proofs are based on intuitions regarding why a result holds. While the proofs that resulted from the shotgun strategy in our study can be described as 
"slick", the proofs that resulted from the targeted strategy tended to be conceptually rich.

\section{3. 2. Member-checking interviews}

We were unable to contact Frank to participate in our member-checking interviews, but we asked Theodore and Sarah the following question: "I noticed that you usually spent time reading and getting familiar with a problem and formulating a plan for how to construct a proof. Once you settled on a plan you tended to stick with it until you produced a proof. Do you think that's a fair description?" Their responses confirmed our interpretation:

Sarah: I mean yes, but I'm not sure how other people do it.

Theodore: Yes Interviewer: Okay, Always? Do you know where that comes from? Can you tell me a little bit more about that?

Theodore: Well for one thing. I learned that you're solving word problems. Like let's say high school simple problems, but word problems. I basically had a method where I have to translate the words into mathematical equations. So I go slowly through the problem and for each sentence you find the piece of information and you translate that, you know. And that's like, I'm tutoring right so this is what I try to tell my students to get down every single piece of information to get to a mathematically useful piece of information. And once you have translated all the information then you can go ahead and compute or whatever.

When asked if this was something that they ordinarily did, both Sarah and Theodore again agreed. Additionally, Theodore's member-checking interviews provided insights regarding the motivations for his targeted strategy.

Theodore: Okay I heard a story once. They said that they came up with some math problems. This was from some other study. They presented these problems to both college students and professors. And they said that the pattern they saw was is that the students would try right away to tackle the problem but the professors would sit back and like try to understand the problem. So I'm just saying what I said before, I think a lot of people have their instinct to just rush right into it without truly understanding what's going on. Interviewer: And so you try to control and do the opposite and sort of try to be very measured and come up with a plan before proceeding?

Theodore: Yeah. ${ }^{2}$

Here Theodore described his behavior as an attempt to emulate the practice of

\footnotetext{
2 It is possible that Theodore was describing Schoenfeld's (1985) seminal Mathematical Problem-solving, but we have no way of knowing what specific study Theodore was referencing, or even if Theodore was aware of the specific study himself.
} 
mathematicians.

\section{3. 3. Avoiding impasses}

Proof construction efforts that used the targeted strategy were consistent with the first three stages of Polya's (1957) problem-solving model; students would understand the task, choose a plan, and implement a plan. These students exhibited less of an inclination to monitor the implementation of their plans, sometimes sticking with a plan that we (as observers) recognized as unproductive. (For instance, consider Theodore trying to prove that $g$ " $(x)>0$ in the excerpt in 4.2.2). Nonetheless, students implementing the targeted strategy were generally able to avoid "chasing wild mathematical geese" by carefully choosing productive plans that were likely to succeed from the outset, since they did not begin implementing a plan until they felt they had a full understanding of the task.

\section{Discussion}

\section{1. Summary of main results}

This study explored the proving behaviors of six highly successful mathematics students. We observed that there was substantial variation in the strategies they used to write proofs. The targeted/shotgun distinction was introduced in this work to account for this variation. This distinction points to two different strategies for successful proof construction. The shotgun strategy involves moving between multiple plans in an attempt to find an efficient solution. The targeted strategy involves understanding the task well, using this understanding to see why the result holds, and then formalizing this understanding.

\section{2. Limitations of our findings and future research questions}


In this paper, we sought to explore approaches that could successfully be employed to solve proving tasks in undergraduate mathematics. We did not intend to engage in sample-to-population generalization. That is, we do not have grounds to claim that highly successful students all make use of these proving approaches. Research with a larger population of successful students would be needed to address these claims.

A related question concerns the proving behavior of less successful students. Do ordinary or struggling mathematics majors attempt to use a shotgun strategy or a targeted strategy, but do so less successfully than the students described in this paper? Or do they approach proving tasks in other ways? If less successful students $d o$ use a shotgun or targeted strategy unsuccessfully, why is this the case? Are there specific resources that they lack in applying these strategies? We observe that using a shotgun strategy involves quickly generating many plausible ways to prove a theorem, which likely involves having “applicability conditions" for when an approach is useful or recognizing similarities between the statement being proven and statements proven in the past. Using a targeted strategy involves the ability to formalize intuitive (possibly graphical) arguments. If students are unable to use such strategies, perhaps it is because they lack these competencies.

An additional question concerns how the constraints of this study and undergraduate mathematics, specifically asking students to prove statements in a relatively short period of time, affected students' strategy use. With regard to the shotgun strategy, from our observations, the proofs that students are asked to write in undergraduate mathematics tend to be relatively short. There are several good reasons for this: Students have such difficulty with other aspects of mathematics (e.g., setting up a 
proof framework, reasoning from definitions) that writing short proofs is challenging enough for students, so assessments requiring longer proofs would simply achieve floor effects (i.e., almost all students will get these longer tasks wrong so it will not be useful in differentiating student ability in terms of grades). Further, longer proofs may take a long time to construct, perhaps hours or days. Hence, assessing students' ability to write these may be infeasible, particularly in research contexts. However, one consequence of having students only write short proofs is that these proofs can often be written by applying a standard technique. One can therefore often succeed in undergraduate mathematics simply by developing a repertoire of standard techniques and being able to apply these techniques quickly. In this sense, the shotgun strategy might not be successful when one moves on to more advanced areas of mathematics, where tasks cannot be solved by applying known techniques. Such responses were evident in Chase's member checking interview and also in a study of Duffin and Simpson (2006), who reported that many graduate students needed to shift from brute force to meaning making approaches to succeed in graduate mathematics.

In the same vein, translating an informal argument into a proof can be a timeconsuming process, even for those who are experienced proof writers (cf., Samkoff, Lai, \& Weber, 2012). The time constraints in this study, and in most undergraduate mathematics assessments, might push students away from a potentially time-consuming targeted strategy and toward a shotgun strategy, which has the potential to pay quicker dividends.

\subsection{Relating the shotgun and targeted strategies to models of problem-solving}

When working on a novel proving task there is a tension between the process of 
choosing a plan and the process of determining that an impasse has been reached with the current plan. The targeted strategy places emphasis on choosing a plan. This is facilitated by a relatively lengthy getting to know the task phase, in which the provers carefully choose a plan based on their developing understanding of the result to be proven. The effort placed into choosing an initial plan, means that most of the plan evaluation effort essentially occurs before the initial plan is chosen. Thus, with the targeted strategy, provers tend to spend a significant amount of time exploring each plan they choose.

In contrast, the shotgun strategy emphasizes whether impasses have been reached and deemphasizes the process of choosing which plan to try next. This is facilitated by a large collection of approaches relevant to the task at hand and a low threshold for when a plan can/should be abandoned. The relatively small amount of time invested in choosing the current plan means that it can also be quickly abandoned whenever progress wanes.

Problem-solving models such as Carlson and Bloom's (2005) include both orienting/planning stages, where a prover gets to know the task at hand and chooses an initial plan, and checking stages, where a prover evaluates whether an impasse has been reached. Other problem-solving models have roughly equivalent elements (e.g. Polya, 1957; Schoenfeld, 1985). The important contribution of our work is in illustrating that, at least with the highly successful mathematics majors in this study, these two types of activities are linked. Emphasis on getting to know the task and carefully choosing a plan reduces the need for abandoning that plan, and reduces the need for frequent evaluations of whether an impasse has been reached. Similarly, de-emphasis of the getting to know the task stages of problem-solving, increases the need for frequent evaluations of whether an impasse has been reached and lowers the threshold for what should be considered an 
impasse. In other words, the shotgun/targeted strategy distinction helps illustrate that there is a relationship between the stages of problem-solving models, where increasing emphasis on one stage reduces the emphasis on another. This suggests that problemsolving models cannot be applied uniformly across all provers, since some stages of these models are more relevant to particular proofs and provers than others.

Additionally, the shotgun/targeted distinction highlights that there is more than one way that undergraduates can succeed at proving, at least within the domain of undergraduate mathematics and timed tests. In this sense, there might not be a single problem-solving model that describes the proving behaviors of undergraduate students. This is consistent with findings that mathematicians approach proof-related tasks in fundamentally different ways (e.g., Pinto \& Tall, 1999; Burton, 2004; Weber, 2013; Weber, Inglis \& Mejía-Ramos, 2014).

\subsection{Relevance to teaching}

This work highlights that successful students may implement, and perhaps have preferences for, vastly different proof construction strategies and that these students exist side-by-side in undergraduate mathematics classrooms. Since our data does not consist of students with both shotgun and targeted strategy preferences in the same classroom we are unable to make empirical claims regarding what the identification of these two strategies implies for teaching. However, our member-checking data as well as Duffin and Simpson's (2006) study suggest that the shotgun strategy may be less effective for graduate level mathematics. So we tentatively claim that specific discussion of how shotgun vs. targeted strategy choice can or should relate to task context may be beneficial for both graduate students and advance undergraduates who work on difficult proofs. 


\section{Acknowledgments}

This material is based upon the work supported by the National Science Foundation under grant DRL-1008641. Any opinions, findings, and conclusions or recommendations expressed in this material are those of the authors and do not necessarily reflect the views of the National Science Foundation.

\section{References}

Alcock, L. J. \& Simpson, A. P. (2004). Convergence of sequences and series: Interactions between visual reasoning and the learner's beliefs about their own role. Educational Studies in Mathematics, 57(1),1-32.

Anderson, J., Boyle, C., \& Yost, G. (1986). The geometry tutor. Journal of Mathematical Behavior, 5, 5-20.

Burton, L. L. (2004). Mathematicians as enquirers: Learning about learning mathematics. Springer: Dordrecht.

Cahill, D. J. (1997). Target marketing and segmentation: Valid and useful tools for marketing. Management Decision, 35(1), 10-13.

Carlson, J. A. (2010). Avoiding traps in member-checking. Qualitative Report, 15(5), 1102-1113.

Carlson, M. P. (1999). The mathematical behavior of six successful mathematics graduate students: Influences leading to mathematical success. Educational Studies in Mathematics, 40(3), 237-258.

Carlson, M. P., \& Bloom, I. (2005). The cyclic nature of problem-solving: An emergent multidimensional problem-solving framework. Educational Studies in Mathematics, 58(1), 45-75.

DeFranco, T. C. (1996). A perspective on mathematical problem solving expertise based on the performance of male Ph.D. mathematicians. In J. Kaput, A. H. Schoenfeld, \& E. Dubinsky (Eds.), Research in Collegiate Mathematics Education, II (pp. 195-213). CBMS Issues in Mathematics Education, Vol. 6. Providence, RI: American Mathematical Society.

Duffin, J. \& Simpson, A. (2006). The transition to independent graduate studies in mathematics.Issues in Mathematics Education, 13, 233-246. 
Firestone, W. (1993). Alternative arguments for generalizing from data as applied to qualitative research. Educational Researcher, 22, 16-23.

Furinghetti, F., \& Morselli, F. (2007). For whom the frog jumps: The case of a good problem solver. For the Learning of Mathematics, 27(2), 22-27.

Garuti R., Boero P. \& Lemut E. (1998). Cognitive unity of theorems and difficulty of proof. Proceedings of the 22nd PME Conference, Stellenbosh, South Africa, 2, 345 - 352.

Gibson, D. (1998). Students' use of diagrams to develop proofs in an introductory real analysis. Research in Collegiate Mathematics Education, 2, 284-307.

Harel, G., \& Sowder, L. (1998). Students' proof schemes: Results from exploratory studies. In A. H. Schoenfeld, J. Kaput, \& E. Dubinsky (Eds.), Research in collegiate mathematics education III (pp. 234-283). Providence, RI: American Mathematical Society.

Harel, G., \& Sowder, L. (2007). Toward comprehensive perspectives on the learning and teaching of proof. Second handbook of research on mathematics teaching and learning, 2, 805-842.

Hart, E. (1994). A conceptual analysis of the proof writing performance of expert and novice students in elementary group theory. In J. Kaput, and Dubinsky, E. (Ed.), Research issues in mathematics learning: Preliminary analyses and results (pp. 49-62). Washington: Mathematical Association of America.

Iannone, P. \& Inglis, M. (2010). Self-efficacy and mathematical proof: Are undergraduates good at assessing their own proof production ability? In Proceedings of the $13^{\text {th }}$ Conference for Research in Undergraduate Mathematics Education. Raleigh, North Carolina.

Ko, Y. Y., \& Knuth, E. (2009). Undergraduate mathematics majors' writing performance producing proofs and counterexamples about continuous functions. The Journal of Mathematical Behavior, 28(1), 68-77.

Lockwood, E., Ellis, A. B., Dogan, M. F., Williams, C., \& Knuth, E. (2012). A framework for mathematicians' example-related activity when exploring and proving mathematical conjectures. In Proceedings of the 34th Annual Meeting of the North American Chapter of the International Group for the Psychology of Mathematics Education (pp. 151-158).

Malone, J., Douglas, G., Kissane, B., \& Mortlock, R. (1980). Measuring problem-solving. In S. Kruklik (ed.) NCTM 1980 yearbook: Problem-solving in school mathematics. Washington, D.C. NCTM.

Mejia-Ramos, Weber, K., \& Fuller, E. (in press). Factors influencing students' propensity for semantic and syntactic reasoning in proof writing: A case study. To appear in International Journal of Research in Undergraduate Mathematics Education. 
Moore, R. C. (1994). Making the transition to formal proof. Educational Studies in Mathematics, 27(3), 249-266.

Pinto, M., \& Tall, D. (1999). Student constructions of formal theory: Giving and extracting meaning. In O. Zaslavsky (Ed.), Proceedings of the 23rd Conference of the International Group for the Psychology of Mathematics Education (Vol. 4, pp. 65-73). Haifa, Israel: PME.

Polya, G. (1957). How to Solve It: a new aspect of mathematical method, ed. London: Penguin.

Raman, M. (2003). Key ideas: What are they and how can they help us understand how people view proof? Educational Studies in Mathematics, 52, 319-325.

Reif, F. (2008). Applying cognitive science to education: Thinking and learning in scientific and other complex domains. MIT Press: Cambridge, MA.

Samkoff, A., Lai, Y., and Weber, K. (2012). On the different ways that mathematicians use diagrams in proof construction. Research in Mathematics Education, 14(1), 49-67.

Sandefur, J., Mason, J., Stylianides, G. J., \& Watson, A. (2013). Generating and using examples in the proving process. Educational Studies in Mathematics, 83(3), 323-340.

Savić, M. (2011). Where is the logic in student-constructed proofs. In Proceedings of the 14th Conference on Research in Undergraduate Mathematics Education (Vol. 2, pp. 445-456).

Schoenfeld, A. H. (1980). Teaching problem-solving skills. American Mathematical Monthly, 794-805.

Schoenfeld, A. H. (1985). Mathematical Problem-solving. Orlando, FL: Academic Press.

Schoenfeld, A. H. (1987). What's all the fuss about metacognition? In A. H. Schoenfeld (Ed.), Cognitive science and mathematics education (pp. 189-215). Hillsdale, NJ: Lawrence Erlbaum Associates.

Selden, J., \& Selden, A. (1995). Unpacking the logic of mathematical statements. Educational Studies in Mathematics, 29(2), 123-151.

Selden, A., \& Selden, J. (2008). Overcoming students' difficulties in learning to understand and construct proofs. In M. Carlson \& C. Rasmussen (Eds.), Making the Connection: Research and Practice in Undergraduate Mathematics. (pp. 95-110). MAA: Washington, DC.

Tall, D., \& Vinner, S. (1981). Concept image and concept definition in mathematics with particular reference to limits and continuity. Educational studies in mathematics, 12(2), 151169.

VanSpronsen, H. D. (2008). Proof processes of novice mathematics proof writers. Ann Arbor, MI: ProQuest LLC.

Weber, K. (2001). Student difficulty in constructing proofs: The need for strategic knowledge. Educational Studies in Mathematics, 48(1), 101-119. 
Weber, K. (2005). Problem-solving, proving, and learning: The relationship between problemsolving processes and learning opportunities in the activity of proof construction. The Journal of Mathematical Behavior, 24(3), 351-360.

Weber, K. (2006). Investigating and teaching the thought processes used to construct proofs. Research in Collegiate Mathematics Education, 6, 197-232.

Weber, K. (2013). On the heterogeneity of mathematical practice with respect to proof. In M. Fried \& T. Dreyfus (Eds.) Mathematicians and mathematics educators: Searching for common ground. Springer: Dordrecht.

Weber, K., \& Alcock, L. (2004). Semantic and syntactic proof productions. Educational Studies in Mathematics, 56(2-3), 209-234.

Weber, K., \& Alcock, L. (2005). Using warranted implications to understand and validate proofs. For the Learning of Mathematics, 34-51.

Weber, K. \& Alcock, L. J. (2009). Proof in advanced mathematics classes: Semantic and syntactic reasoning in the representation system of proof. In D.A. Stylianou, M.L. Blanton \& E. Knuth (Eds.), Teaching and Learning Proof Across the Grades: A K-16 Perspective (pp. 323-338). New York, NY: Routledge.

Weber, K., Inglis, M., \& Mejía-Ramos, J.P. (2014). How mathematicians obtain conviction: Implications for mathematics instruction and research on epistemic cognition. Educational Psychologist, 49, 36-58.

Zandieh, M., Roh, K. H., \& Knapp, J. (2014). Conceptual blending: Student reasoning when proving "conditional implies conditional" statements. The Journal of Mathematical Behavior, 33, 209-229.

Zazkis, D., Weber, K., \& Mejia-Ramos, J.P. (2014). Activities that mathematics majors use to bridge the gap between informal arguments and proofs. Proceedings of the Conference for Psychology of Mathematics Education. Vancouver, Canada. 


\section{APPENDIX: Tasks}

1: Suppose $f(0)=f^{\prime}(0)=1$. Suppose $f^{\prime \prime}(x)>0$ for all positive $x$. Prove that $f(2)>2$.

2: Prove that the only real solution to the equation $x^{3}+5 x=3 x^{2}+\sin x$ is $x=0$.

3: Suppose $f(x)$ is a differentiable even function. Prove that $f^{\prime}(x)$ is an odd function.

4: Prove that $a^{2}+a b+b^{2} \geq 0$ for all real numbers $a$ and $b$.

5: Suppose $f^{\prime \prime}(x)>0$ for all real numbers $x$. Suppose $a$ and $b$ are real numbers with $a<b$. Define $g(x)$ as the line through the points $(a, f(a))$ and $(b, f(b))$. Prove that for all $x \in[a, b], f(x) \leq g(x)$.

6: Prove that $\int_{-a}^{a} \sin ^{3}(x) d x=0$ for any real number $a$.

7: Let $f$ be differentiable on $[0,1]$, and suppose that $f(0)=0$ and $f^{\prime}$ is increasing on $[0,1]$. Prove that $g(x)=\frac{f(x)}{x}$ is increasing on $(0,1)$. 